\title{
Analyzing the Effects of Geometrical and Particle Size Uncertainty in Circulating Fluidized Beds using CPFD Simulation
}

\author{
Janitha C. Bandara $^{1} \quad$ Britt M.E. Moldestad $^{1} \quad$ Marianne S. Eikeland $^{1}$ \\ ${ }^{1}$ Department of Process, Energy and Environmental Technology, University of South-eastern Norway, \\ Norway \\ \{janitha.bandara, britt.moldestad, Marianne.Eikeland\}@usn.no
}

\begin{abstract}
Computational fluid dynamic modeling and simulation is becoming a useful tool in the detailed analysis of multiphase flow systems. The level of uncertainty is different depending on selected modeling concept and numerical schemes. Physical uncertainties originated from geometrical dimensions and particle properties are important aspects. In this work, CPFD method was used to analyze the effect of dimensional uncertainty of loopseal pipe diameter and particle size distribution in a circulating fluidized bed. Five different pipe diameters were studied and $20 \%$ growth in particle circulation rate was observed as the diameter reduced from $30 \mathrm{~mm}$ to $26 \mathrm{~mm}$. The effect of small changes in the particle size distribution was negligible and the particle circulation rate decreased by $32 \%$ with monodisperse particles of mean size.
\end{abstract}

Keywords: CPFD simulation, uncertainty, circulating fluidized bed, particle circulation rate

\section{Introduction}

Computational fluid dynamic (CFD) modeling and simulation is extensively used in designing and optimizing of reactive and non-reactive systems. The flow predictions for single phase systems with CFD is very much precise and however, multiphase systems are still encountering number of theoretical and numerical challenges, such as wide range of spatio-temporal scales (length scales between single particles, particle clusters, computational grid and geometry), collision, shear and interact of particles, mass and momentum exchange between phases (Pannala et al., 2011).

Different techniques have been developed to model the multiphase systems. Direct numerical simulation (DNS) requires the least modeling effort. However, the computational time is high as it resolves the complete flow field around each particle of the system and the particle movement is modeled with Newton's equation of motion (Bale et al. 2019; Tang et al. 2016). Lattice-Boltzmann method (LBM) uses less computational power as the flow field around the particle is approximated by lattice-Boltzmann equation (Qi , Kuang, and Yu 2019). The simulation time can further be reduced by discrete element method (DEM) which averages the fluid flow in the scale of computational grid. The particle collisions are modeled using the soft-sphere or hard-sphere approach. Even with increasing computer power, DEM simulations are predicted to be not viable in the coming decade for commercial scale reactors. The contact detection of particles and calculating geometric areas of contact consume more than $80 \%$ of the computational time in DEM. The Eulerian-Eulerian modeling which is also identified as the two-fluid (TFM) or multi-fluid modeling has been the main interest over decades due to its capacity of modeling large-scale systems. TFM requires high modeling effort as the particle phase is also considered as a fluid and the properties are derived using the kinetic theory of granular flow (KTGF). Difficulties and complexities of including the particle size distribution, loss of discrete nature of the particles, numerical (false) diffusion are the major disadvantages related to TFM. The computer efficiency of the DEM can be improved by using probabilistic strategy for the particle contact modelling such as the multiphase particle in cell (MP PIC) method where the collisional forces are derived as a stress gradient in the Eulerian grid (Ma \& Zhao, 2018; Moliner et al., 2018; Pannala et al., 2011). The model complexity increases progressively from DNS to TFM and simultaneously, the uncertainty also increases due to excess use of empirical correlations, assumptions, approximations and averaging.

The sensitivity, uncertainty and errors are three aspects of the CFD predictions. The sensitivity is primarily involved with the computational grid and convergence test should be performed in first hand for the CFD simulations. The time step and number of computational particles (MP PIC method) are other sensitivity tests. Sensitivity of different coefficients, models and model constants used have equal importance, which can also be addressed as uncertainties (Ostermeier et al., 2019). The uncertainties have different dimensions related to (Mathelin et al., 2005; Walters \& Huyse, 2002):

- Assumptions in the main model (i.e. incompressible, inviscid, linearization, neglecting temperature dependences of coefficients) 
- Deficiency of information related to phenomenological or auxiliary models (i.e. turbulence model, reaction kinetics)

- Discretization and computational errors (i.e. flux approximation scheme, round off, iterative)

- Describing the physical reality (the geometry, initial and boundary conditions, particle properties such as size distribution, density and shape)

The fundamental structure of the conservation equation are fixed in many CFD codes. The suitability of different numerical schemes and optimization of involved auxiliary models and model parameters are possible. However, uncertainties originated from physical reality should be minimized in first hand. Some experimental data suffers from lack of supporting information (i.e. reporting of mean size of particles over size distribution, the pipe diameters without mentioning internal or external etc.).

Circulating fluidized bed (CFB) technology is a widely applied industrial process. Robust control over the particles, high heat and mass transfer rates are the basic advantages of the CFB technology. The simplest arrangement of a CFB system is illustrated in Figure 1 and certain CFB systems can be consisted with two or multiple reactors. A typical system contains a bedding material that circulates in a closed loop without being removed out from the system. Riser operates in the fast fluidization regime and the particles are carried away with high gas flowrates, which are separated by a cyclone and fed back to the riser via a flow control valve. Mechanical valves suffer from wearing in high temperature applications where non-mechanical devices such as loopseal are highly preferred in the industry. Rate of particle circulation is one of the important parameters in CFB. The dimensions and aeration of the loopseal should be designed and optimized to fit the targeted flow and avoid gas bypass from the riser.

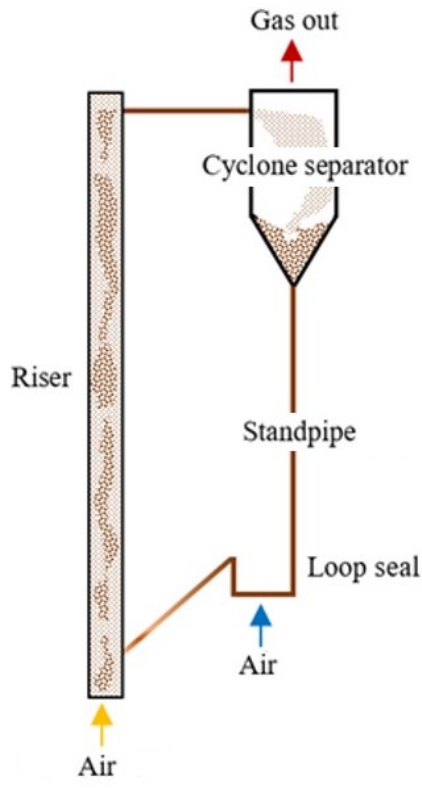

Figure 1: Circulating fluidized bed
The rate of particle circulation is governed by number of parameters such as loopseal aeration, riser gas flow, loopseal dimensions and the particle properties. As discussed prior, deviations in the simulation geometry and particle properties are a subset of the overall uncertainty. This work includes the uncertainty analysis related to pipe dimensions of the loopseal and particle size distribution for MP PIC simulated results. Barracuda VR is a commercial software package built on the MP PIC platform, which brought forward the concept of computational particle fluid dynamics (CPFD). The simulations were performed using Barracuda 17.3.0 and Intel(R) Core(TM) $3.50 \mathrm{GHz}$ processor.

\section{CPFD modeling}

Andrews \& O'Rourke (1996) extended the MP PIC method to particle flow systems, which was developed into CPFD. Later, Snider developed the scheme into three dimensional dense particle flows (Snider, 2001). The subsequent improvements of the particle collision modeling are discussed in several subsequent publications (O'Rourke \& Snider, 2012, 2014; O'Rourke \& Snider, 2010; O'Rourke et al., 2009). The fluid phase is modeled with Navier-Stokes equations, similar to DEM and TFM. The modeling of the particle phase has hybrid characteristics of discrete and continuum modeling. The real particles are grouped into parcels (computational particles) such that the billions of particles can be represented by millions of parcels. A certain parcel contains a number of real particles having same size, density and velocity. The parcel movement through the fluid domain is modeled similar to DEM. The particle collision force is calculated as a stress gradient on Eulerian grid in the advanced time step and mapped back to real time with interpolation functions. Unlike the TFM, the discrete nature of the particles is preserved and the implementation of the particle size distribution is straightforward.

\subsection{Governing equations}

The governing equations are referred from Snider (Snider, 2001). Gas phase mass and momentum conservation are modeled with continuity and time averaged Naiver-Stokes equations:

$$
\begin{aligned}
& \frac{\partial\left(\alpha_{g} \rho_{g}\right)}{\partial t}+\nabla \cdot\left(\alpha_{g} \rho_{g} u_{g}\right)=0 \\
& \frac{\partial\left(\alpha_{g} \rho_{g} u_{g}\right)}{\partial t}+\nabla \cdot\left(\alpha_{g} \rho_{g} u_{g} u_{g}\right)=-\nabla P-F+ \\
& \nabla \cdot\left(\alpha_{g} \tau_{g}\right)+\alpha_{g} \rho_{g} g
\end{aligned}
$$

Where $\alpha_{g}, \rho_{g}$ and $u_{g}$ are gas phase volume fraction, density and velocity respectively. $F$ is the total momentum exchange with particle phase per volume, $g$ is the gravitational acceleration, $\mathrm{P}$ is the pressure, and $\tau_{g}$ 
is the gas phase stress tensor. The stress tensor of the gas phase is given by:

$$
\tau_{g}=\mu_{g}\left[\left(\nabla u_{g}+\Delta u_{g}^{T}\right)-\frac{2}{3} \nabla \cdot u_{g} I\right]
$$

$\mu_{g}$ refers to the shear viscosity that is the sum of the laminar and turbulent components. The large eddy simulation is used for the large-scale turbulence modeling while the subgrid scale turbulence is captured with the Smagorinsky model:

$$
\mu_{g, t}=C_{s} \rho_{g} \Delta^{2}\left|\nabla u_{g}+\Delta u_{g}^{T}\right|
$$

The default value for the model constant $C_{S}$ is $0.01 . \Delta$ is the sub-grid length scale and calculated by:

$$
\Delta=(\delta x \delta y \delta z)^{1 / 3}
$$

The interface momentum transfer is calculated through the viscous drag force:

$$
F=\iint f\left\{m_{p}\left[D_{p}\left(u_{g}-u_{p}\right)-\frac{\nabla P}{\rho_{p}}\right]\right\} d m_{p} d u_{p}
$$

Subscript $\mathrm{P}$ refers to the particle phase properties where $\mathrm{m}$ and $\mathrm{u}$ symbolizes the mass and velocity respectively. $D_{p}$ is the drag function. The particle phase dynamics are derived using the particle distribution function (PDF) calculated from the Liouville equation given as:

$$
\frac{\partial f}{\partial t}+\nabla\left(f u_{p}\right)+\nabla u_{p}\left(f A_{p}\right)=0
$$

Where $A_{p}$, is the particle acceleration and is expressed by:

$$
A_{p}=\frac{\partial\left(u_{p}\right)}{\partial t}=D_{p}\left(u_{g}-u_{p}\right)-\frac{\nabla P}{\rho_{p}}-\frac{\nabla \tau_{p}}{\rho_{p} \alpha_{p}}+g
$$

$\alpha_{p}$ is particle volume fraction and $\tau_{p}$ is particle stress function used to formulate the interphase interactions of particles.

$$
\begin{aligned}
& \alpha_{p}=\iint f \frac{m_{p}}{\rho_{p}} d m_{p} d u_{p} \\
& \tau_{p}=\frac{10 P_{s} \alpha_{p}^{\beta}}{\max \left[\left(\alpha_{c p}-\alpha_{p}\right), \varepsilon\left(1-\alpha_{p}\right)\right]}
\end{aligned}
$$

$P_{S}$ is a constant with the units of pressure, $\alpha_{c p}$ is the particle volume fraction at close packing, $\beta$ is a constant between 2 and 5 and $\varepsilon$ is a very small number on the order of $10^{-7}$.

\section{Computational method}

The experimental data of Thapa et al (2016) was used for the comparison of simulation results. The loopseal and riser pipe diameters were $30 \mathrm{~mm}$ and $50 \mathrm{~mm}$ respectively. The system pressure and rate of particle circulation were available where the circulation rate had been measured by interrupting (stopping) the loopseal aeration followed by measuring the time to accumulate a certain volume of particles at the standpipe. Sand with of $2650 \mathrm{~kg} / \mathrm{m}^{3}$ in density and $130 \mathrm{~mm}$ in mean diameter (size distribution from $50 \mathrm{~mm}$ to $250 \mathrm{~mm}$ ) was the particle phase. Air at atmospheric pressure and temperature was the loopseal and riser aeration fluid

The fluid volume was developed using SOLIDWORKS 2018 and imported to Barracuda VR 17.3.0. Uniform grid option was used and the grid refinement at narrow sections was needed to capture the geometry domain accurately. The total number of cells in the domain was 467376. The turbulence was modelled with large eddy simulation and the partial donor cell method (a weighted average method of central difference and upwind schemes) was used as the

\begin{tabular}{|c|c|c|}
\hline & Parameter & Value \\
\hline [1] & $\begin{array}{l}\text { Closed pack volume } \\
\text { fraction }\end{array}$ & 0.6 \\
\hline [2] & $\begin{array}{l}\text { Maximum momentum } \\
\text { redirection }\end{array}$ & $40 \%$ \\
\hline [3] & $\begin{array}{l}\text { Particle-wall collision } \\
\text { (Normal \& tangential) }\end{array}$ & 0.85 \\
\hline [4] & Diffuse bounce & 3 \\
\hline [5] & $\begin{array}{l}\text { Pressure constant in } \\
\text { particle stress model }\left(\mathrm{P}_{\mathrm{s}} \text { in }\right. \\
\text { Pascal) }\end{array}$ & 5 \\
\hline [6] & $\begin{array}{l}\text { Initial time step } \\
\text { (seconds) }\end{array}$ & 0.0003 \\
\hline
\end{tabular}
advection numerical scheme. The default values were used for the number of iterations, residuals and the minimum and maximum values of Courant-FriedrichsLewy (CFL) parameter (which satisfy the convergence criteria). Values of the model constants and other simulation parameters are given in Table $\mathbf{1}$.

Table 1: Simulation parameters

As the particle flow pattern of a circulating fluidized bed with loopseal is analysed, the riser operates at dilute phase while the loopseal at dense phase. Further, the fluid drag is a function of the particle volume fraction. Therefore, the Wen-Yu-Ergun drag model (Gidaspow) was used as the Ergun correlation has been extensively validated for dense systems. The Wen-Yu model is used at higher gas volume fractions than 0.8 , which is given by (Gidapow, 1994): 


$$
\begin{gathered}
D_{\text {Wen-Yu }}=\frac{3}{8} C_{d} \frac{\rho_{g}}{\rho_{p}} \frac{\left|u_{g}-u_{p}\right|}{r_{p}} \alpha^{-2.65} \\
C_{d}=\quad \frac{24}{R e}, \quad(R e<0.5) \\
C_{d}=\frac{24}{R e}\left(1+0.115 R e^{0.687}\right), \quad(0.5 \leq R e \leq 1000)
\end{gathered}
$$

As the gas volume fraction decreases below 0.8 , the Ergun correlation is used,

$$
D_{\text {Ergun }}=0.5\left(\frac{C_{1} \alpha_{p}}{\alpha_{g} R e}+C_{2}\right) \frac{\rho_{g}}{\rho_{p}} \frac{\left|u_{g}-u_{p}\right|}{r_{p}}
$$

The default values for the laminar and turbulent coefficients in the Barracuda VR are $180\left(\mathrm{C}_{1}\right)$ and 2.0 $\left(\mathrm{C}_{2}\right)$ where those are 150 and 1.75 in original Ergun formulation. The particle Reynolds number is given by:

$$
R e=\frac{2 \rho_{p}\left|u_{g}-u_{p}\right|}{\mu_{g}}\left(\frac{3 V_{p}}{4 \pi}\right)^{1 / 3}
$$

The rate of particle circulation was measured by the flux plane implemented at the overflow pipe. A flux plane stores the data of the amount of particles by species and fluid pass across a defined area. Two additional flux planes were positioned at standpipe and riser to recognize the steady state conditions. The computational domain was initially occupied exclusively with air where a particle feed flow boundary was used to introduce the particles into the system. The "particle feed control" option was linked to the particle flow boundary to maintain the particle mass in the system between $0.58 \mathrm{~kg}$ and $0.60 \mathrm{~kg}$ throughout the entire simulation time. The number density manual at the particle feed was set to 200 , which decides the resolution of computational particles in the domain. The boundary conditions, flux planes and the pressure monitoring locations are illustrated in Figure 2.

The loop seal exerts the highest resistance for the particle flow. Therefore, the pipe diameter of the loopseal was gradually reduced from $30 \mathrm{~mm}$, which is the measured value from a scaled drawing, to $27 \mathrm{~mm}$ in successive simulations. The pipe diameters given in the sketch can be the outside diameter and hence the actual diameter for the fluid volume should be equal or less than $30 \mathrm{~mm}$.

Further, the particle size distribution can have uncertainties. Therefore, results from the reported distribution was compared with two other size distributions. The first two alternatives were taken from the arbitrary assumption that the exact size distribution can bias more towards smaller sizes than the reported value (if the sieving had not been done sufficiently). The other set up considered the mono sized particles with mean diameter of 125 microns. The size distributions are plotted in Figure 3.

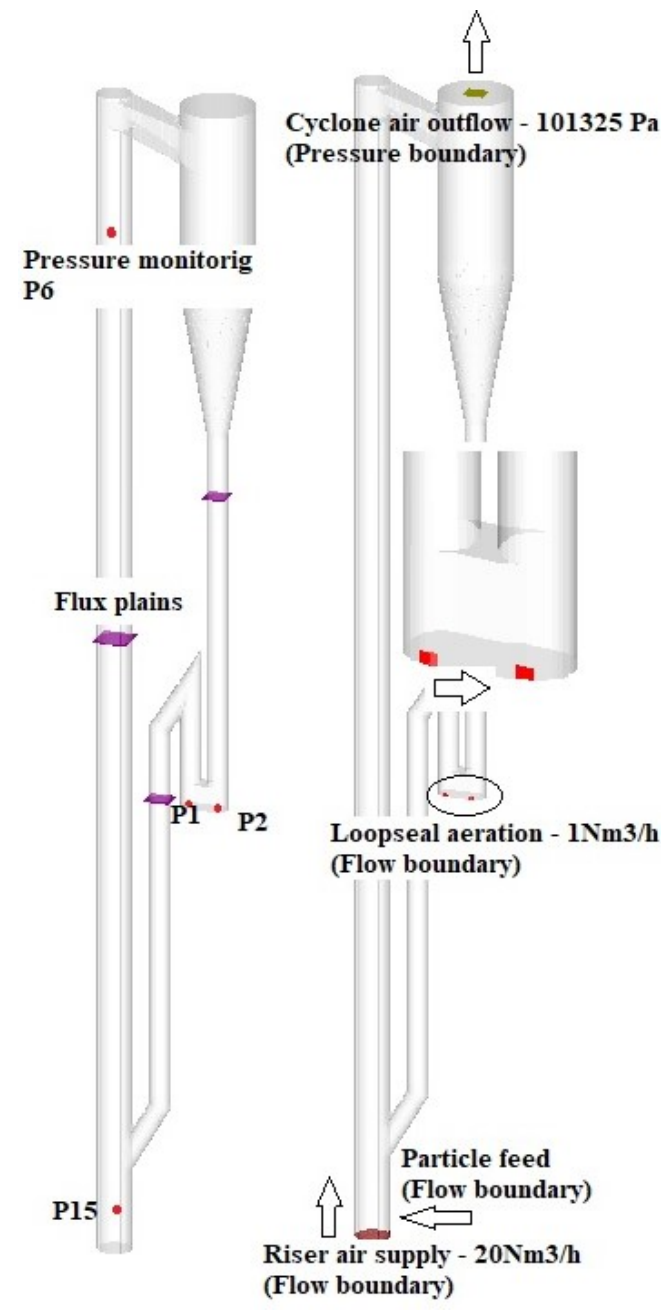

Figure 2: Computational domain; boundary conditions, flux plane and pressure transient data points

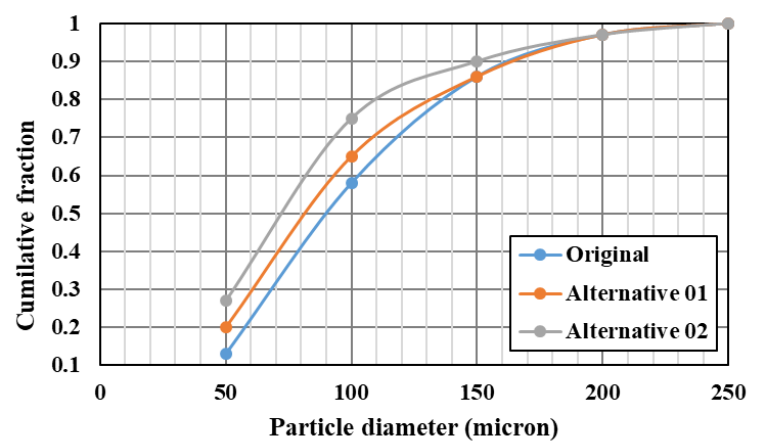

Figure 3: Particle size distribution

\section{Results and Discussion}

The simulation results for the original geometry was compared with the experimental data of Thapa et al, based on the rate of particle circulation and the system pressure. The particle distribution over the circulating fluidized bed at steady state operation (pseudo) is depicted in Figure 5. The enlarged sections in Figure 5 shows the particle flow behavior in the loop seal. 
The particle volume fractions over the riser and the cyclone sections are below 0.1 where a dense particle region can be observed at the bottom of the cyclone conical section. The particle volume fraction in the standpipe is approximately 0.5 , which reduces in the horizontal section and recycle pipe. With the observed bubbles, the standpipe seems to operate at bubbling fluidization regime. A rigorous fluidization is prevailed at the horizontal section and the recycle pipe, which indicates that a large fraction of the loopseal aeration passes through the recycle pipe and ends up in the riser. In the loopseal, air tends to flow near the walls of the side of aeration and further, the airflow does not penetrate much within particle bed (in the direction of injection). Extended grid refining at the air injection may improve the length of penetration and however, large differences in the grid sizes (in all $\mathrm{X}, \mathrm{Y}$ and $\mathrm{Z}$ directions) are not preferred in CPFD. Further, a grid cell should be sufficient to place several parcels.

The particle circulation rate was averaged over 30 seconds during steady state operation and calculated to be $315 \mathrm{~kg} / \mathrm{h}$, which is approximately equal to the experimental data. However, the rate was highly dynamic and large fluctuations between $100 \mathrm{~kg} / \mathrm{h}$ to $1000 \mathrm{~kg} / \mathrm{h}$ could be observed. The airflow rate across the flux plane was $-0.00037 \mathrm{~kg} / \mathrm{s}$ and the loopseal aeration was $0.00036 \mathrm{~kg} / \mathrm{s}$. This guarantee the proper operation of the loopseal that does not allow gas to bypass from riser via loopseal. Further, small amount of riser gas is recycled back across loopseal without escaping from the cyclone top. This is possible at high particle circulation rate, where the air is carried along with the voidage of the particle phase. The gas flow across the loopseal had not been monitored during experimental studies and the simulation results are useful in further optimizing the loopseal dimensions. The system pressure was also monitored at different locations (given in Figure 2) and the results are given in Figure 4 together with experimental data. Pressure prediction from the simulation is lower than the experimental data over the entire system. The cyclone exhaust pipe was replaced with a pressure boundary with $101325 \mathrm{pa}$ (atmospheric pressure) boundary value and the downstream pressure drop was excluded. Geometrical lengths, pressure monitoring locations, particle size distribution and the assumption of spherical particles can be other physical uncertainties for the deviated pressure readings.

Olatunde, and Fasina (2019) have mentioned the observed deviations related to coefficients of Ergun equation for different particles. The laminar viscous coefficient has reported to as high as 267 while the turbulent coefficient up to 4.02 . The barracuda default values of 180 and 2 were used in this study. Further, the competency of the Wen \& Yu model for the dilute phase systems is not concretely validated as Ergun model. The particle hold up in the riser depends on the drag force exerted by the gas flow. In this case, the Wen \& Yu model might over predict the drag force and consequently caused a reduced particle, which could lead for a lower pressure drop in the riser. This has a direct effect on the reduced pressure reading from the simulation at the recycle chamber. The effect of the particle phase modelling parameter of closed pack volume fraction is also significant for the pressure drop in dense particle regions. Due to the lack of data related to particle phase, the default value of 0.6 was used. The prediction error of the system pressure can be originated from one or many of these uncertainties and lack of data.

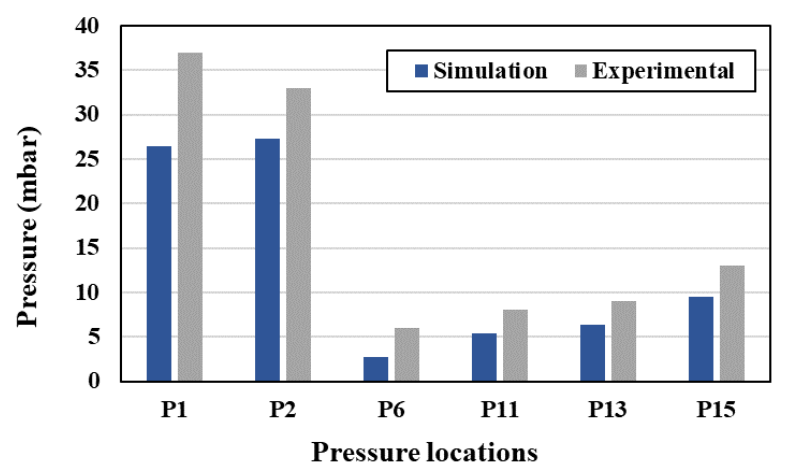

Figure 4: System pressure: experimental vs simulation

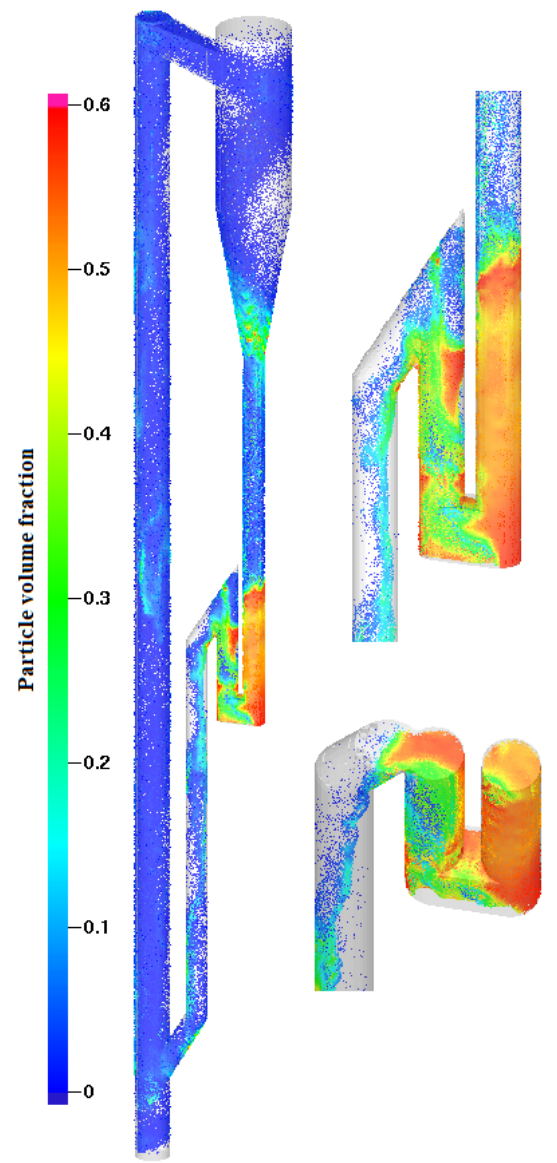

Figure 5: Particle volume fractions over CFB at steady state 


\subsection{Effect of the loopseal pipe diameter}

Successive updates of the simulation parameters related to particle properties were needed to achieve the particle circulation rate similar to experimental values and the optimized values used are given in Table 1. However, the system pressure was not considerably sensitive for the analyzed parameters, where the observed deviations might originate from errors and uncertainties. The highest deviation of the pressure was recorded at the loopseal, which was lower than experimental data. Hence, simulations were performed for different diameters of the loopseal piping.

As illustrated in Figure 6, the particle bed height at the standpipe is slightly increasing towards reduced diameters. The loopseal balances the cumulative pressure drop of the remaining sections of the CFB system and the bed height at the standpipe is automatically adjusted following the system variations.

The rate of particle circulation and the system pressure are illustrated in Figure 7 and Figure 8 respectively. The particle circulation rate is greatly influenced by the pipe diameter, which showed a $20 \%$ increment when the diameter was reduced from $30 \mathrm{~mm}$ to $27 \mathrm{~mm}$. The variation shows second order polynomial characteristics against pipe diameter. The gas velocity across the loopseal increases as the diameter is reduced and consequently, the fluid drag force on the particle increases. Similarly, the air bypass from cyclone to riser across loopseal has also increased.

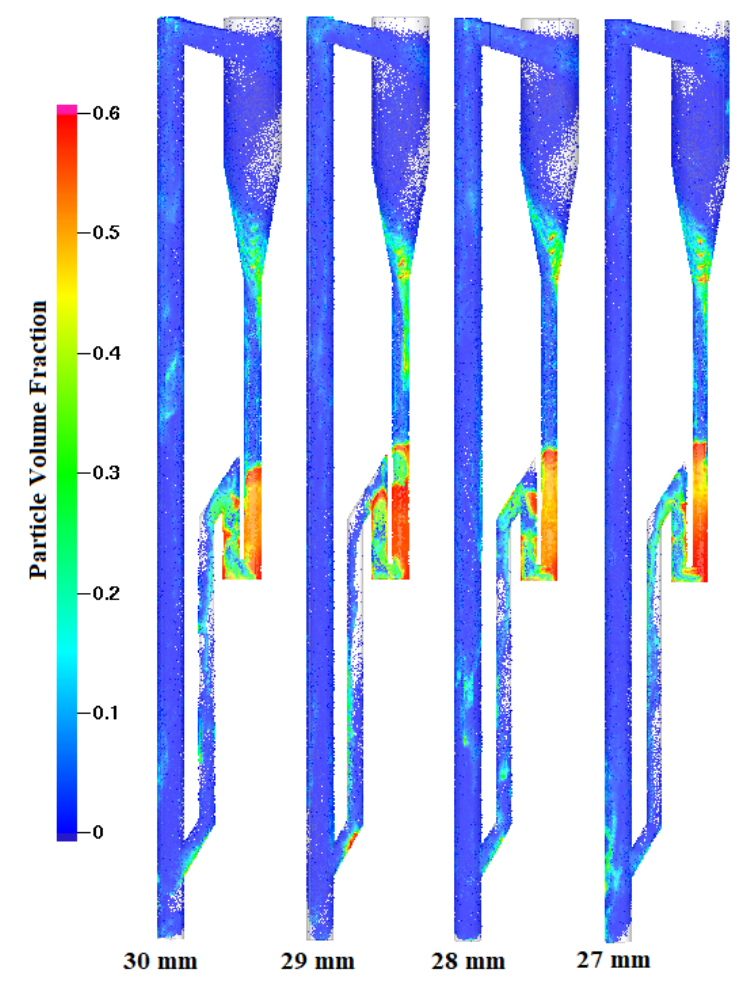

Figure 6: Particle flow hydrodynamics at different loopseal pipe diameters

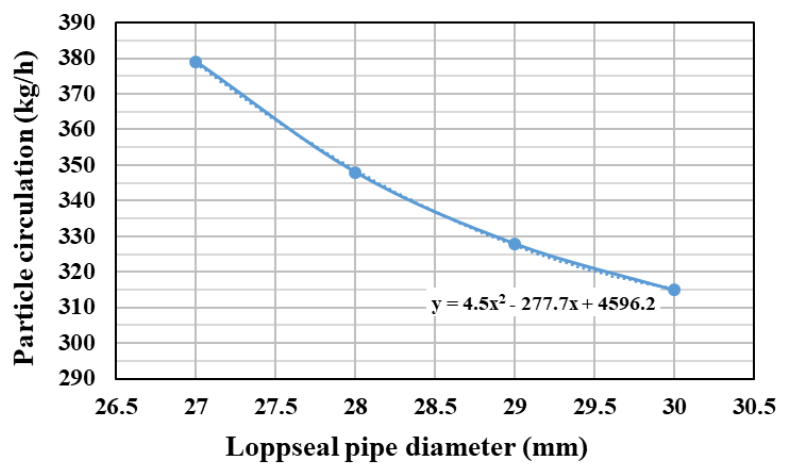

Figure 7: Change of particle circulation rate over different loopseal pipe diameters

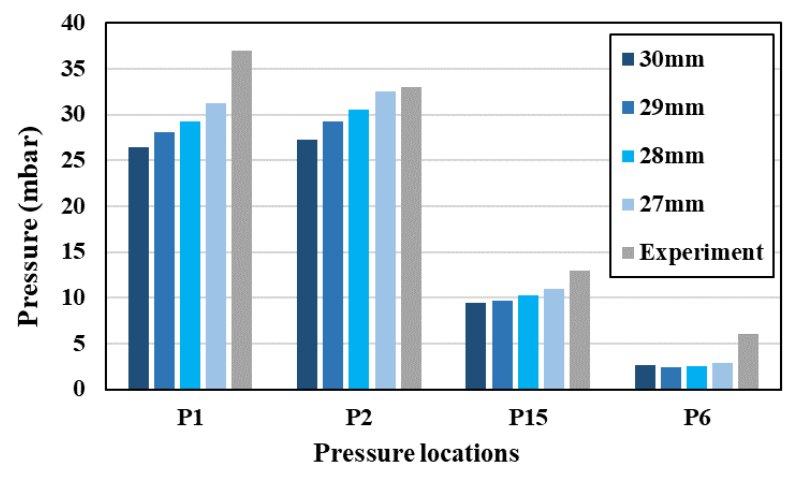

Figure 8: Change of system pressure over different loopseal pipe diameters

An improvement in the pressure prediction can be observed and the results reach the experimental values at $\mathrm{P} 2$ and P15. The particle holdup within the riser compartment can be high at increased particle circulation, which contribute for increased pressure at the riser bottom, P15. Prediction error of pressure at P1 may be originated from incorrect height of the recycle pipe, where the height effect can be further analyzed.

\subsection{Effect of the particle size distribution}

Particle size distribution is measured using sieving analysis equipment. Inadequate sieving time may avoid sufficient separation of particles. Further, with the difficulties of implementing the particle size distribution, monodisperse particles have been widely used (i.e. two-fluid modelling). Therefore, the simulation results from different size distributions as given in Figure $\mathbf{3}$ and 125 micron monodisperse particles were compared using original geometry. The rate of particle circulation is given in Figure 9.

Significant changes of particle circulation was not observed between different size distributions used. Specially, merely the weight fractions were changed keeping the smallest and largest particle size similar to original. However, a considerable reduction of particle circulation, about 32\%, was observed with monodisperse particles. This is a clear illustration of the force exerted by smaller particles on larger particles and 
highlights the percentage error related to using mean size rather than size distribution. The geometry and the particle mass were equal for all cases and further, the loopseal operates at fluidizing regime (i.e. size distribution can affect the pressure drop at packed bed conditions), which can be the reason behind the similar pressure results for different size distributions.

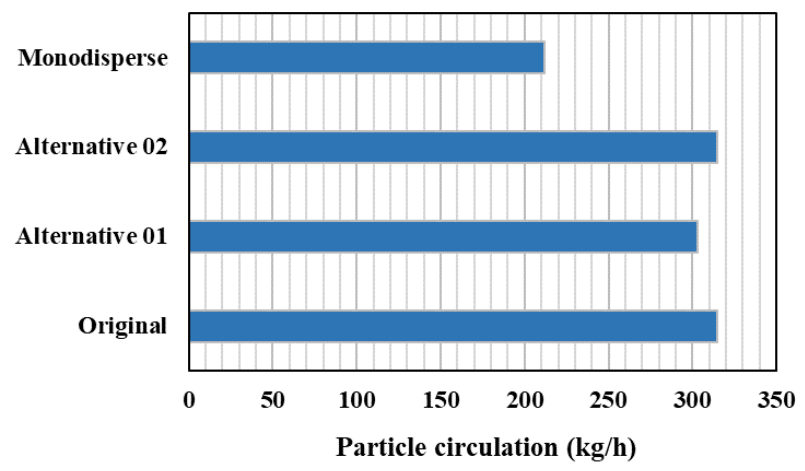

Figure 9: Change of particle circulation with size distribution

\section{Conclusion}

This work was carried out to analyze the effect of selected uncertainties related to geometrical lengths and particle size distribution in a circulating fluidized bed system. If the experiments are deliberately designed to generate data for CFD model validation, all the necessary parameters are available. However, whenever the existing experimental data from the literature are used in validation, certain uncertainties can be existed and therefore, adequate illustrations should be presented to compensate. The uncertainties related to physical reality and all accurately measurable parameters should be minimized (avoided) prior to the sensitivity analysis of models and model constants.

The loopseal pipe diameter displayed a great influence over particle circulation rate. The system pressure prediction was lower than experimental data in all the sections of the domain. Prediction error of the pressure at the recycle pipe was comparatively high, which might originate from incorrect height of computational geometry used or deviated pressure at the riser bottom due to inaccurate particle holdup in the riser. Small changes in the particle size distribution within the same smallest and largest sizes did not cause much change in particle circulation rate. However, monodisperse particles with mean particle size gave a substantially reduced circulation rate, which was $32 \%$ lower.

More uncertainties can be prevailed related to geometrical lengths of other sections, particle mass in the system, particle properties such as sphericity and closed pack volume fraction, loopseal aeration velocity and location. Therefore, further analysis will be supportive to demonstrate the effect of mentioned uncertainties and it is highly recommended to perform specially designed experiments for CFD model validation with all the required data.

\section{Acknowledgements}

The authors like to forward their gratitude to Rajan Thapa for sharing his experimental data and University of South-eastern Norway for facilitating with computer resources and Barracuda VR CPFD software package.

\section{References}

M. J. Andrews and P. J. O'Rourke. The multiphase particlein-cell (MP-PIC) method for dense particulate flows. International Journal of Multiphase Flow, 22: 379-402, 1996.

S. Bale, S. S. Tiwari, K. Nandakumar, and J. B. Joshi. Effect of Schmidt number and $\mathrm{D} / \mathrm{d}$ ratio on mass transfer through gas-solid and liquid-solid packed beds: Direct numerical simulations. Powder Technology, 354: 529-39, 2019.

D. Gidapow. Multiphase Flow and Fluidization: Continuum and Kinetic Theory Descriptions. Boston: Americal Press, 1994.

H. Ma and Y. Zhao. Investigating the fluidization of disklike particles in a fluidized bed using CFD-DEM simulation. Advanced Powder Technology, 29:2380-2393, 2018.

L. Mathelin, M. Y. Hussaini, and T. A. Zang. Stochastic approaches to uncertainty quantification in CFD simulations. Numerical Algorithms, 38: 209-236, 2005.

C. Moliner, F. Marchelli, N. Spanachi, A, Martinez-Felipe, B. Bosio, and E. Arato. CFD simulation of a spouted bed: Comparison between the Discrete Element Method (DEM) and the Two Fluid Model (TFM). Chemical Engineering Journal, 2018

G. Olatunde and O. Fasina. Modified Ergun Equation for Airflow through Packed Bed of Loblolly Pine Grinds. KONA Powder and Particle Journal, advpub, 2019.

P. J. O'Rourke and D. M. Snider. Inclusion of collisional return-to-isotropy in the MP-PIC method. Chemical Engineering Science, 80: 39-54, 2012.

P. J. O'Rourke and D. M. Snider. A new blended acceleration model for the particle contact forces induced by an interstitial fluid in dense particle/fluid flows. Powder Technology, 256: 39-51, 2014.

P. J. O'Rourke and D. M. Snider. An improved collision damping time for MP-PIC calculations of dense particle flows with applications to polydisperse sedimenting beds and colliding particle jets. Chemical Engineering Science, 65: 6014-6028, 2010.

P. J. O'Rourke, P. Zhao, and D. M. Snider. A model for collisional exchange in gas/liquid/solid fluidized beds. Chemical Engineering Science, 64: 1784-1797, 2009.

P. Ostermeier, S. DeYoung, A. Vandersickel, S. Gleis, and H. Spliethoff, H. (2019). Comprehensive investigation and comparison of TFM, DenseDPM and CFD-DEM for dense fluidized beds. Chemical Engineering Science, 196: 291-309, 2019. 
Pannala, S., Syamlal, M., \& O'Brien, T. J. (2011). Computational Gas-Solids Flows and Reacting Systems: Theory, Methods and Practice. Hershey, USA: Engineering Science Reference.

Qi, Z., S. Kuang, and A. Yu. 2019. Lattice Boltzmann investigation of non-Newtonian fluid flow through a packed bed of uniform spheres. Powder Technology 343; 225-36.

Snider, D. M. (2001). An Incompressible Three-Dimensional Multiphase Particle-in-Cell Model for Dense Particle Flows. Journal of Computational Physics, 170, 523-549.

Tang, Y., Y. M. Lau, N. G. Deen, E. A. J. F. Peters, and J. A. M. Kuipers. 2016. Direct numerical simulations and experiments of a pseudo-2D gas-fluidized bed. Chemical Engineering Science 143; 166-80.

Thapa, R. K., Frohner, A., Tondl, G., Pfeifer, C., \& Halvorsen, B. M. (2016). Circulating fluidized bed combustion reactor: Computational Particle Fluid Dynamic model validation and gas feed position optimization. Computers \& Chemical Engineering, 92, 180-188.

Walters, R. W., \& Huyse, L. (2002). Uncertainty Analysis for Fluid Mechanics with Applications. In. Hanover: NASA Center for AeroSpace Information (CASI). 